Part II has eight chapters on advanced dynamical theory, and deals with the details of intensities and properties of field amplitudes inside the crystal. It points out how Kato's important contributions to sphericalwave treatment justify, for the most part, our use of the simpler but somewhat unrealistic plane-wave approach and points out some cases where the latter treatment fails. In most cases, intensity predictions by the plane-wave theory are virtually the same as in the spherical-wave treatment, but in the particular situation of overlapping wave fields, phase-shift-predicted fringes in certain Pendellösung experiments are incorrect. This led to errors in the precise measurement of atomic scattering factors, but Kato's spherical-wave theory correctly explains the results. The more general treatment of the theory by Takagi is presented in Chapter 11. It covers the general case of any incident-wave type, plane or spherical, and is particularly useful for elucidating wave propagation in highly distorted crystals. This section is completed by a chapter on wave tracing and includes the author's seminal work explicitly demonstrating the existence of several branches of the dispersion surface in the two-beam case. In all, this part is an extensive review of the sophistication of the propagation of X-ray Bloch waves in perfect crystals and will be an important resource for the advanced researcher.

Part III has two chapters presenting a detailed treatment of wave propagation in moderately and heavily deformed crystals. It covers many topics, with experimental results from the Paris group (A. Authier, Y. Epelboin, C. Malgrange), and the efforts of U. Bonse, A. Lang, N. Kato, J. R. Patel, D. Taupin and others to study dynamical diffraction effects in these highly deformed crystals and to use the theory to study defects such as stacking faults, growth defects, precipitation, and propagation through and reflection from crystals with externally applied strains.

Part IV has three chapters and covers the coupling of applications of dynamical diffraction to the special properties of synchrotron radiation, providing an important resource for the experimentalist. It addresses crystal optics, topography, standing-wave techniques for atom location, Fresnel zone plates and recent investigations of refractive lenses. Professor Authier covers many of these topics in sufficient depth to give the reader more than a general understanding and he provides up-to-date references that enable one to pursue any of these topics in even greater depth.
Professor Authier has worked on this book over the many years of his dedication to the understanding of the diffraction of $\mathrm{X}$ rays from perfect and nearly perfect crystals. From my point of view, it is a tour de force. In 1941, von Laue's book Roentgenstrahlen Interferenzen was the seminal treatment for the first 30 years of dynamical diffraction. Professor Authier's book brings us up to the present with a second such treatment of enormous range. He brings us from the nascent period to a description of the field in its most vigorous state. He has given us a historic sense of the evolution of dynamical diffraction, exposed for us its mathematical complexities and shown us how its applications are at the forefront of modern research in solid-state physics and materials science. The book is mandatory reading for specialized workers in dynamical diffraction, and it is an essential reference for anyone interested in modern applications of X-ray scattering using synchrotron radiation.

\section{Boris W. Batterman}

Advanced Light Source

Lawrence Berkeley Laboratory

Berkeley

CA 94720

USA

E-mail: bwb1@cornell.edu

\section{Diffuse neutron scattering from crystal-}

line materials. By Victoria M. Nield and David A. Keen. Pp. xvi + 317. Oxford: Oxford University Press, 2000. Price

GBP 69.50. ISBN 0-19-851790-4.

In this book, the authors have two stated aims. The first is: 'to make diffuse scattering data interpretation more tractable'; the second is: 'to provide an overview of the wide range of available interpretative methods, giving enough information for the reader to select the most useful tool for understanding his or her diffuse data, whilst avoiding elaborate and, to the non-specialist, possibly bemusing detail on any specific methodology'. I believe the authors have been admirably successful in meeting these aims. The book is an accessible and comprehensive guide to diffuse neutron scattering and, though written as an introduction to the technique for graduate scientists, will be a valuable reference text for any crystallographer keen to understand and apply modern interpretative techniques to diffuse scattering data. Though the emphasis of the book is undoubtedly on diffuse neutron scattering, with numerous practical examples taken from neutron studies, there is very little material that is not equally of interest to anyone working with $\mathrm{X}$-rays or electrons. The extensive bibliographies at the end of each chapter provide a comprehensive guide to the literature on all aspects of diffuse scattering.

The first chapter gives a brief historical review of the development of interest in diffuse scattering from its earliest beginnings, with the work of such famous names as Debye and Brillouin, to modern times, with current interest extending into protein crystallography. This chapter puts into perspective the special role of neutrons, as opposed to X-rays. The second chapter develops the theory of the basic scattering process specific to neutrons but, even here, $\mathrm{X}$-ray workers will be able to benefit from seeing the differences and similarities in the comparable X-ray scattering theory. The third chapter deals with the theory of diffuse scattering and summarizes the different approaches that have been used to describe it. Important real-space concepts such as short-range-order parameters, size-effect and Huang scattering parameters are described, as well as alternative approaches founded in reciprocal-space descriptions. Again, in this chapter there is very little that is not equally applicable to X-ray scattering. Chapters 4 and 5 deal, respectively, with details of neutron-scattering data collection and data reduction. They provide a succinct description of the different kinds of instruments used and the information that can be obtained from them. Most importantly, reactor and spallation neutron sources are compared, with implications for the types of experiment that can be performed, providing a useful guide for the would-be experimenter. Chapter 6 details how analytical approaches to understanding and analysing diffuse scattering have been progressively replaced by various computer simulation and modelling techniques which allow more complex systems to be studied. Valuable introductions are given to molecular dynamics (MD), Monte Carlo (MC), reverse Monte Carlo (RMC) and the pair distribution function (PDF) methods. The remaining chapters describe, in varying degrees of detail, studies of systems of great diversity. Chapters 7 and 8 deal primarily with the more traditional subjects of substitutional disorder in alloys and simple oxides, and progress to more complex topics, including magnetic systems, quasicrystals and systems that include solvated hydrogen. The special ability of neutrons to see hydrogen and magnetic systems is emphasized. The final chapters concentrate on 


\section{book reviews}

three specific areas prominent in recent years. Chapter 9 deals with superionic conductors including silver iodide and various fluorite-related phases, Chapter 10 considers molecular systems including ice, $\mathrm{C}_{60}$, plastic phases, phases of various solidified gases, and Chapter 11 discusses framework structures, particularly in the context of the silica polymorphs.

My only slight criticism is that the book ends rather abruptly! Perhaps this is because the topic is one that is very much ongoing and the book relates only the 'story so far'. A brief chapter devoted to possible future developments and directions would have nicely rounded off what is otherwise a really excellent volume.

\section{T. R. Welberry}

Research School of Chemistry

Australian National Laboratory

Canberra

ACT 0200

Australia

E-mail: welberry@rsc.anu.edu.au

Phonons in nanostructures. By Michael A. Stroscio and Mitra Dutta. Pp. 288.

Cambridge: Cambridge University Press, 2001. Price GBP 65.00. ISBN

0521792797 .

During the last fifteen years, M. Stroscio has devoted his scientific activity to the understanding of phonon physics in low-dimensional structures. He recently collected his notes and with his wife, M. Dutta, has synthesized them into this comprehensive monograph on the latest developments in the theory of crystal dynamics in semiconductor nanostructures. The presentation is clear and pedagogic, with detailed and elegant mathematical derivations from fundamental principles and wave equations to special expressions of phonon equations in quantum-confined semiconductor structures. Non-experts will be delighted by the organization of the book, which starts with basic considerations on semiconductor crystals and leads them through general topics on crystal dynamics of bulk materials, progressively, to the theory of phonon physics in nanoscale systems. For each section, a long list of relevant references is appended. Special attention is paid to the influence of dimensionality on electronphonon interaction in zinc-blende and wurtzite crystal structures, and the corresponding scattering rates in quantum wells, quantum wires and quantum dots. In passing, specific issues on non-equilibrium phonons and phonon generation in nanostructures are discussed. The final two chapters are devoted to the role of confined phonons in nanoscale semiconductor optics and, more specifically, to the stimulating topic of phonon engineering in nanostructures, with the ultimate goal of controlling dissipation and boosting the performances of electronic and optoelectronic devices.

Overall, this book is appropriate for researchers and graduate students in physics, engineering, materials science and chemistry, who have an interest in solid-state technology.

\section{Jean-Pierre Leburton}

Beckman Institute

University of Illinois at Urbana-Champaign

405 North Mathews Avenue

Urbana

IL 61801

USA

E-mail: jleburton@uiuc.edu

\section{books received}

The following books have been received by the Editor. Brief and generally uncritical notices are given of works of marginal crystallographic interest; occasionally, a book of fundamental interest is included under this heading because of difficulty in finding a suitable reviewer without great delay.

Symmetry and structure. Edited by J. Boeyens. New York: Elsevier Science, 2001. Crystal Engineering, special issue, Vol. 4, Nos. 2-3, pp. 61-291. Price not available. ISSN 1463-0184. Selected proceedings of the IUCr Workshop, Indaba III, 2000, Skukuza, South Africa. Contains an introduction by the editor and 15 additional contributions. 